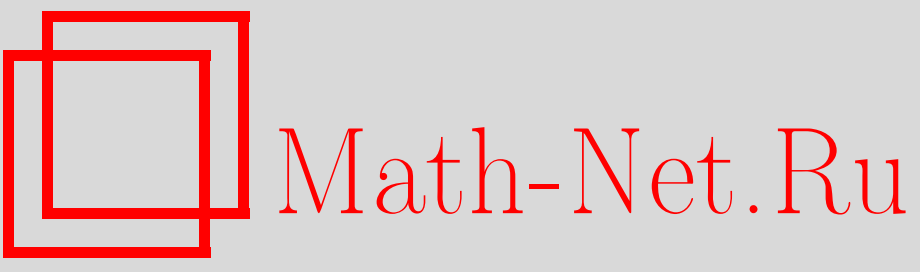

А. Б. Жеглов, О классификации двумерных локальных тел. II, УМН, 2000, том 55, выпуск 6, 135-136

DOI: https://doi.org/10.4213/rm342

Использование Общероссийского математического портала Math-Net.Ru подразумевает, что вы прочитали и согласны с пользовательским соглашением

http://www.mathnet.ru/rus/agreement

Параметры загрузки:

IP : 54.197 .130 .99

26 апреля 2023 г., $15: 47: 37$ 


\title{
О КЛАССИФИКАЦИИ ДВУМЕРНЫХ ЛОКАЛЬНЫХ ТЕЛ. II
}

\author{
А.Б. ЖЕГлов
}

1. В работе [1] была приведена классификация двумерных локальных тел в случае, когда канонический автоморфизм имеет бесконечный порядок, и в случае, когда тело расщепимо, канонический автоморфизм имеет конечный порядок, тело вычетов является полем и характеристика тела равна нулю.

В настоящей работе мы изучаем расщепимые двумерные локальные тела, у которых канонический автоморфизм имеет конечный порядок и характеристика тела не равна нулю. Как следствие мы получаем некоторые результаты, касающиеся строения группы Брауэра двумерного локального поля (исчерпьвающее изложение почти всех известных на настоящий момент результатов о строении группы Брауэра гензелевых полей можно найти в [2], а также в [3]). В частности, это положительный ответ на гипотезу о равенстве экспоненты и индекса произвольного тела из группы Брауэра $C_{2}$-поля (см., например, $\left.[2 ; \S 3.4 .5]\right)$ в случае, когда $C_{2}$-поле имеет вид $k((u))((t)), k$ - алгебраически замкнуто.

Напомним некоторые определения из [1].

ОПРЕДЕЛЕнИЕ 1 . Пусть $K$ и $k$ - произвольные тела. Будем говорить, что $K$ является $n$-мерным локальным телом, имеющим тело $k$ последним телом вычетов, если тело $K$ имеет следующую структуру. Или $n=0$ (и при этом $K=k$ ), или $K$ обладает дискретным нормированием $\nu: K^{*} \cup\{0\} \rightarrow \mathbb{Z} \cup\{\infty\}$, где $\nu: K^{*} \rightarrow \mathbb{Z}$ - сюръективный гомоморфизм, $\nu(0)=\infty$ и $\nu(a+b) \geqslant \inf (\nu(a), \nu(b)) ;$ и его кольцо нормирования $\mathscr{O}:=\{x \in K: \nu(x) \geqslant 0\}$ является полным и отделимым относительно топологии, задаваемой $\nu$, и его тело вычетов является $(n-1)$-мерным локальным телом с последним телом вычетов $k$.

ОПРЕДЕЛЕНИЕ 2. Изоморфизмом локальных тел $K$ и $K^{\prime}$ будем называть изоморфизм их как колец, сохраняющий нормирования $\nu, \bar{\nu}$, где $\bar{\nu}-$ дискретное нормирование тела $\bar{K}$.

Произвольное двумерное локальное тело будем называть расщепимым, если существует гомоморфизм $\bar{K} \hookrightarrow \mathscr{O} \subset K$, являющийся сечением отображения $\mathscr{O} \rightarrow \mathscr{O} / \wp=\bar{K}$.

Элементы $z \in \mathscr{O}, \nu(z)=1$, и $u \in \overline{\mathscr{O}} \subset \bar{K}, \bar{\nu}(u)=1$, будем назьвать локальными параметрами (или переменными) тела $K$.

Рассмотрим кольцо некоммутативных полиномов от двух переменных $\mathbb{Z}\langle\alpha, \delta\rangle$. Определим отображение $\sigma: \mathbb{Z}\langle\alpha, \delta\rangle \rightarrow \mathbb{Z}\left\langle\alpha, \delta, \delta_{i} ; i \geqslant 1\right\rangle$ со значениями в колњце некоммутативных многочленов от переменных $\alpha, \delta, \delta_{i} ; i \geqslant 1$, как отображение, которое заменяет в каждом слове, начиная с правого конца, сочетания типа $\delta^{i} \alpha$ на $\delta_{i}$, т.е. $\sigma\left(\alpha^{a_{1}} \delta^{b_{1}} \ldots \alpha^{a_{n}} \delta^{b_{n}}\right)=\alpha^{a_{1}} \delta_{b_{1}} \ldots \delta_{b_{n-1}} \alpha^{a_{n}-1} \delta^{b_{n}}$, где $a_{1}, b_{n} \geqslant 0, a_{i}, b_{j} \geqslant 1, i>1, j<n,-$ натуральные числа.

В колце $\mathbb{Z}\langle\alpha, \delta\rangle$ определим многочлены $S_{i}^{k}$ как сумму всех мономов, принадлежащих орбите монома $\underbrace{\alpha \ldots \alpha}_{i-k} \underbrace{\delta \ldots \delta}_{k}$ под действием группы подстановок $S_{i}: S_{i}^{k}=\sum_{\tau \in S_{i} / G} \tau(\underbrace{\alpha \ldots \alpha}_{i-k} \underbrace{\delta \ldots \delta}_{k})$, где $G$ - стационарная подгруппа.

Пусть $K$ расшепимо. Фиксируем некоторые параметры $z$ и $u$. Тогда имеет место

ПрЕДЛОЖЕнИЕ $1 . K=\bar{K}((z))$ как векторное пространство, закон умножения двух рядов из которого задается с помощью тождества $z a=a^{\alpha} z+a^{\delta_{1}} z^{2}+a^{\delta_{2}} z^{3}+\cdots$, где $a \in \bar{K}, \alpha$ - некоторый автоморфизм, $\delta_{i}: \bar{K} \rightarrow \bar{K}$ - некоторые линейнье отображения, удовлетворяющие тождеству:

$$
\delta_{i}(a b)=\sum_{k=0}^{i} \sigma\left(\delta^{i-k} \alpha\right)(a) \sigma\left(S_{i}^{k} \alpha\right)(b), \quad a, b \in \bar{K} .
$$

Работа выполнена при частичной финансовой поддержке Российского фонда фундаментальных исследований (грант № 96-15-96050) и фонда INTAS (грант № 93-2805). 
СлЕДСтвИЕ 1. Пусть $\alpha=$ Id. Тогда

$$
\delta_{i}(a b)=\delta_{i}(a) b+\sum_{k=1}^{i} \delta_{i-k}(a) \sum_{\left(j_{1}, \ldots, j_{l}\right)} C_{i-k+1}^{l} \delta_{j_{1}} \ldots \delta_{j_{l}}(b)
$$

Здесь $\delta_{0}=\alpha, 0<l \leqslant \min \{i-k+1, k\}, j_{m} \geqslant 1, \sum j_{m}=k$, вектор $\left(j_{1}, \ldots, j_{l}\right)$ принадлежит орбите челочисленного вектора с суммой координат, равной $k$, под действием группь подстановок $S_{l}$.

ЗАмечАниЕ. Предложение справедливо для расщепимого тела произвольной характеристики с произвольным (некоммутативным) телом вычетов. Все нижеследуюшие результаты являются преимущественно его следствиями.

2. Пусть $\bar{K}$ - поле и $z$ - параметр тела $K$. Отображение $\phi: K \mapsto K, \phi(x)=z x z^{-1}$, переводит в себя $\mathscr{O}$ и $\wp$, т.е. дает автоморфизм $\alpha$ поля $\bar{K}$. Он не зависит от выбора $z$.

Теорема 1. Пусть $K$-расщепимое двумерное локальное тело, $\operatorname{char} K=p>0, \bar{K}$ поле, последнее поле вычетов $k=\overline{\bar{K}} \subset Z(K), \alpha=\mathrm{id}$.

Тогда тело $K$ является конечномерныцм над своим иентром тогда и только тогда, когда $K$ изоморфно телу вида $k((u))((z))$ с соотношением $z^{-1} u z=u+x z^{i}$, где $x \in \bar{K}{ }^{p}$, $(i, p)=1$.

СледСтвиЕ 2. В условиях теоремы если $K$ конечномерно над своим иентром, то его индекс ind $K=p$.

СледСтвиЕ 3. Пусть выполнены все условия теоремы, кроме условия $\alpha=\mathrm{id}$. Пусть $\alpha$ имеет конечный порядок, а $K$ - конечномерное тело индекса $p^{k}$.

Тогда либо $K$ ииклично, либо $\alpha=\mathrm{id}$ и $K$ имеет вид, описанный в теореме.

СледСтвиЕ 4. Пусть $F=F_{0}\left(\left(t_{1}\right)\right)\left(\left(t_{2}\right)\right)$ - двумерное локальное поле, $F_{0}$ - алгебраически замкнутое поле. Пусть $A \in \operatorname{Br}(F)$ - тело.

Тогда $A \cong B \otimes C$, где $B$ - чиклическое тело, (ind $B, p)=1, C$ - тело индекса $p^{k}$, имеющее вид, описанный предьдущим следствием.

СледСтвиЕ 5. Гипотеза о том, что әкспонента тела в группе Брауәра $C_{2}$-поля равна его индексу, имеет положительное решение в случае поля $F_{0}\left(\left(t_{1}\right)\right)\left(\left(t_{2}\right)\right)$.

ЗАмечАниЕ. Характеристика поля $F_{0}$ в последнем следствии не имеет значения.

Автор хотел бы выразить глубокую признательность своему научному руководителю А.Н. Паршину и Е.В. Цинку за постоянное внимание к работе, а также В.И. Янчевскому за ценные консультации.

\section{СПИСОК ЛИТЕРАТУРЫ}

[1] ЖКеглов А. Б. // УМН. 1999. Т. 54. №4. С. 169-170. [2] Платонов В. П., Янчевский В. И. // Итоги науки и техники. Совр. пробл. матем. Фунд. напр. Т. 77. М.: ВИНИТИ, 1991. C. 144-262. [3] Jacob B., Wadsworth A.// J. Algebra. 1990. V. 128. P. 126-179.

Московский государственный

Принято редколлегией университет им. М. В. Ломоносова 03.10 .2000 